\title{
Local Isotropy Indicator for SAR Image Filtering: Application to Envisat/ASAR Images of the Doñana Wetland (November 2014)
}

\author{
Belén Martí-Cardona, Carlos López-Martínez, Senior Member, IEEE, and Josep Dolz-Ripollés
}

\begin{abstract}
This paper explores a geometrical and computationally simple operator, named Ds, for local isotropy assessment on SAR images. It is assumed that isotropic intensity distributions in natural areas, either textured or nontextured, correspond to a single cover class. Ds is used to measure isotropy in processing neighborhoods and decide if they can be considered as belonging to a unique cover class. The speckle statistical properties are used to determine suitable Ds thresholds for discriminating heterogeneous targets from isotropic cover types at different window sizes. An assessment of Ds as an edge detector showed sensitivities similar to those of the ratio edge operator for straight, sharp boundaries, centered in the processing window, but significantly better sensitivity for detecting heterogeneities during the window expansion in multiresolution filtering. Furthermore, Ds presents the advantage versus the ratio edge coefficient of being rotationally invariant, and its computation indicates the direction of the main intensity gradient in the processing window. The Ds operator is used in a multiresolution fashion for filtering ASAR scenes of the Doñana wetland. The intensities in isotropic areas are averaged in order to flatten fluctuations within cover types and facilitate a subsequent land cover classification. The results show high degree of smoothing within textured cover classes, plus effective spatial adaptation to gradients and irregular boundaries, substantiating the usefulness of this operator for filtering SAR data of natural areas with the purpose of classification.
\end{abstract}

Index Terms-Doñana wetland, geometrical operator, gradual edges, isotropy, SAR image filtering, texture.

\section{INTRODUCTION}

$\mathbf{T}$ HE DOÑANA National Park wetlands are located in southwest Spain and constitute a dynamic landscape [1]. The utility of spaceborne SAR imagery for wetland observation has been widely reported [2], [3]. Flood extent and vegetation development in Doñana were monitored from 2006 to 2010

Manuscript received March 14, 2014; revised July 05, 2014; accepted October 30, 2014. The ASAR data used in this study was provided by the European Space Agency within the frame of a Category 1 User Agreement. The work was supported in part by the Plan Nacional de $\mathrm{I}+\mathrm{D}+\mathrm{i}$ of the Spanish Ministerio de Ciencia e Innovación (Projects CGL2006-02247, CGL200909801, and TEC2011-28201-C02-01) and in part by the Agencia Andaluza del Agua of the Junta de Andalucía.

B. Martí-Cardona is with the Department of Hydraulics, Maritime, and Environmental Engineering, Universitat Politècnica de Catalunya, 08034 Barcelona, Spain (e-mail: belen.marti@upc.edu).

C. López-Martínez is with the Remote Sensing Laboratory, Universitat Politècnica de Catalunya, 08034 Barcelona, Spain (e-mail: carlos.lopez@ tsc.upc.edu).

J. Dolz-Ripollés is with the Institut Flumen, Universitat Politècnica de Catalunya, 08034 Barcelona, Spain (e-mail: j.dolz@upc.edu).

Color versions of one or more of the figures in this paper are available online at http://ieeexplore.iee.org.

Digital Object Identifier 10.1109/JSTARS.2014.2369133 using Envisat/ASAR images acquired in alternated polarization mode, with HH/VV polarization configuration and at seven different incidence angles [4].

The wetland mapping from the ASAR data required filtering the scenes to smooth out backscattering fluctuations owing to speckle and texture. Literature on speckle filtering is vast. Among the simplest methods are the boxcar or median filters, which perform well at smoothing speckle, but blur edges between different land cover types. Lee [5], Frost et al. [6], and Kuan et al. [7] algorithms improve SAR image filtering performance by making use of the statistical properties of speckle [8]. These algorithms use the coefficient of variation (CV) to measure local stationarity of pixel intensities and different degrees of filtering are applied accordingly. They are effective to approximate the terrain's radar cross section in homogeneous and textured targets but, again, might smear edges to some degree.

In order to preserve image structure and border sharpness, geometric criteria were introduced in the filtering process. The aim was to identify edges within the processing window and adapt the filtering neighborhood to them. In this line, Lee [9] proposed the use of gradient operators to detect edges in four directions (up-down, right-left, and diagonals). Touzi et al. [10] adopted a similar approach but used ratio operators, referred to as $\mathrm{r} 2$, more adapted to the multiplicative nature of speckle noise than the gradient operators. Ever since, different authors have successfully combined geometrical criteria based on ratio edge detectors and statistical filters, mostly Lee's and Frost's, to smooth speckle effects while preserving boundary definition in SAR images [11]-[15]. More complex de-speckling approaches considered anisotropic diffusion using partial derivative equation based methods [16], [17], wavelet transforms [18], [19], shearlet transforms [20], [21], or Markov random field models [19], [22], [23].

The operative mapping from the ASAR imagery for the Doñana wetland's management demanded a relatively simple and robust filtering method, oriented at the subsequent segmentation and classification of the images. Single class areas had to be determined in presence of texture and irregular or gentle edges. The above-mentioned methods combining statistical filters and the ratio edge detector are simple and robust. The ratio edge operator is well adapted to spot straight and abrupt edges, but in natural environments, where boundaries take capricious geometries and can be gradual, its effectiveness can be improved by using the Ds operator, as shown in this work. 
The Ds operator was first introduced in [24] for local isotropy assessment on SAR images, and then applied in [25] and [26] for filtering Envisat/ASAR images of the Doñana wetlands. This paper presents a detail analysis of the Ds operator performance in the context of SAR image filtering. The Ds definition is included in Section II-A. Section II-B assesses the Ds sensitivity to local heterogeneities. Its capability to identify the border between targets is compared to that of the $\mathrm{CV}$ and $\mathrm{r} 2$ operators in different scenarios: presence of straight and irregular boundaries, and in the case of spatial gradients. Section III introduces a methodology for the use of the Ds operator as an isotropy indicator in the multiresolution filtering of SAR images. This methodology is applied to Envisat/ASAR images of the Doñana marshes. Results are shown and compared to those for commonly used speckle filters.

\section{PARAmeter DS For Local Isotropy Assessment}

\section{A. Definition}

Equations (1)-(3) define a geometrical operator, named Ds, for local isotropy assessment on SAR images. In these expressions, I stands for pixel value; i, $\mathrm{j}$ denote image row and column; $\mathrm{N}$ represents the processing neighborhood; and $C_{i}, C_{j}$ are the image coordinates of the neighborhood geometrical center

$$
\begin{aligned}
D_{i} & =\frac{\sum_{i} \sum_{j} i \cdot I(i, j)}{\sum_{i} \sum_{j} I(i, j)} \quad \forall(i, j) \in N \\
D_{j} & =\frac{\sum_{i} \sum_{j} j \cdot I(i, j)}{\sum_{i} \sum_{j} I(i, j)} \quad \forall(i, j) \in N \\
D s & =\sqrt{\left(D_{i}-C_{i}\right)^{2}+\left(D_{j}-C_{j}\right)^{2}} .
\end{aligned}
$$

Ds is referred to as geometrical because its definition depends on the pixel values spatial arrangement, so that image windows with identical histograms can have different Ds values. Ds represents the first-order central moment of the processing neighborhood, normalized by the total intensity. More intuitively, Ds represents the distance in pixels between the neighborhood geometrical centroid and what would be the gravity center if the pixel values were masses, referred to as intensity centroid. This distance provides a measurement of the intensities spatial imbalance, so that Ds yields small values for isotropic distributions (where geometrical and intensity centroids would be almost coincident) and large ones when high and low intensities are preferentially clustered in two different segments of the neighborhood.

Some interesting properties of the Ds parameter can be readily drawn from its definition: first, the Ds value is rotationally invariant. Second, the image physical dimensions (intensity and amplitude) are canceled out by the denominator in (2) and (3), so the Ds parameter can be used as an isotropy measure on any image type (e.g., amplitude or intensity). Finally, the vector defined by the neighborhood geometrical and intensity centers, vector $\left(D_{j}-C_{j}, D_{i}-C_{i}\right)$, approximates the direction of the spatial imbalance.

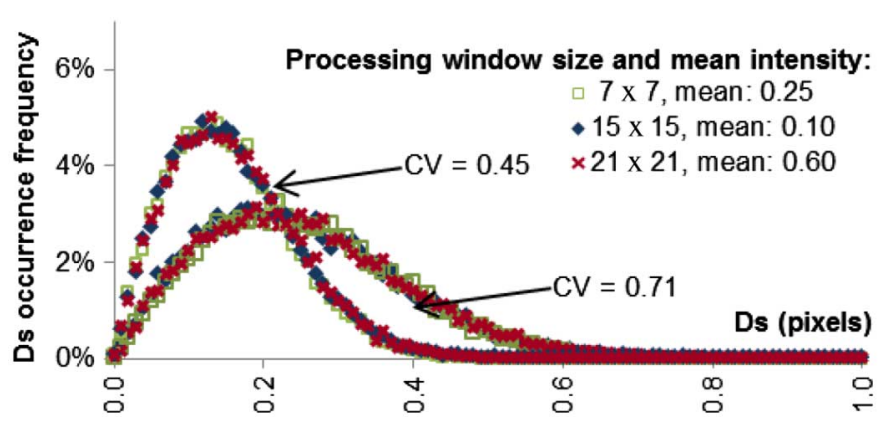

Fig. 1. Ds pdf obtained through Monte Carlo simulations of synthetic SAR images with different sizes and gamma-distributed pixel intensities.

When using Ds for filtering SAR images, the speckle noise stochastic properties are introduced through the thresholds used to discriminate between homogeneous and heterogeneous targets, as discussed in Section II-B.

\section{B. Assessment of Ds as a Heterogeneity Detector}

The probability density function (pdf) of parameter Ds operating over a SAR image of a homogeneous target has been estimated through Monte Carlo simulations. In absence of texture and spatial correlation, the backscattered intensities from a homogeneous target can be modeled by independent realizations of a negative exponential distribution for single-look SAR images, and a gamma distribution when the number of looks is higher than 1 [27]. A synthetic square image was generated so that pixel values were independent realizations of the same gamma distribution (same CV and mean) and the corresponding Ds value was computed through (1)-(3). This process was repeated 20000 times. The occurrence frequency of the Ds values approximates the Ds pdf. Some of the resultant pdfs, which are independent of the mean intensity and window size, are shown in Fig. 1. They depend only on the CV, becoming wider as the $\mathrm{CV}$ increases.

If the synthetic image is generated using other intensity distributions (e.g., Gaussian, negative exponential, and Rayleigh), the Ds pdf turned out to be the same in all cases, as shown in Fig. 2. Hence, it can be said that the Ds pdf over a homogeneous target, meaning by homogeneous that the pixels' intensity can be modeled by independent realizations of the same statistical distribution, does not depend on the distribution type, the mean intensity, nor the processing window size. The Ds pdf is only determined by the pixel values' standard deviation to mean ratio or CV.

The value of Ds over a heterogeneous window comprising pixels of two different gamma distributions was simulated in presence of straight and irregular, sharp and gradual boundaries, and in the case that the edge is or is not centered within the processing window. The simulated edge geometries are depicted in Fig. 3. The edge sensitivity of the Ds, $r 2$, and CV parameters is analyzed in this section for those geometries.

The Ds pdf of a heterogeneous target computed using different window sizes and contrast values is represented in Figs. 4 and 5. It can be observed in these figures that Ds takes clearly larger values over heterogeneous windows than on homogeneous ones, and that it increases with both, window size and 


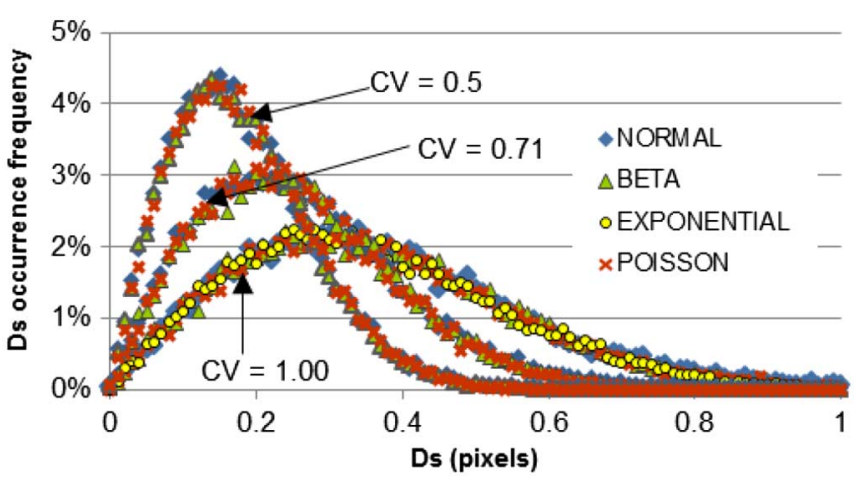

Fig. 2. Ds pdf obtained through Monte Carlo simulations of homogeneous target SAR images, for different pixel intensity distributions.
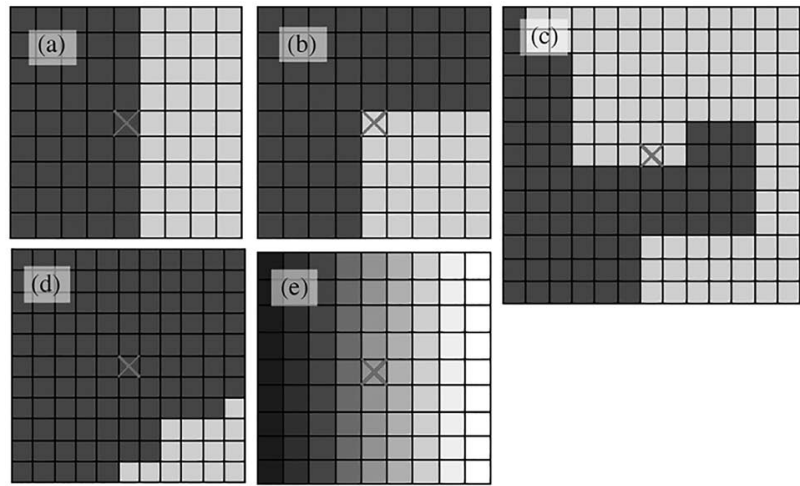

Fig. 3. Simulated edge geometries: (a)-(c) sharp edges passing through the processing window center; (d) not centered sharp edge; (e) gradual edge.

contrast between adjacent targets. Therefore, Ds is sensitive to target heterogeneity within a processing window.

When filtering backscattering fluctuations in a SAR image, it is necessary to test the stationarity of the processing window, so that the computation of filtered values avoids using pixels from different targets. In order to decide on the stationarity of a pixel neighborhood based on the corresponding Ds value, a threshold needs to be chosen, as done with the ratio edge detector or the CV [12]-[14]. To evaluate the performance of Ds as a heterogeneity indicator, the confusion probability has been defined as the average likelihood of misrecognizing a heterogeneous window as homogeneous or conversely. Given a Ds threshold Th, the confusion probability is computed through (4) and graphical interpretation is given by the shaded area in Fig. 4 for threshold 0.37 , window size $7 \times 7$ and contrast 2 .

Given a particular window size and contrast, the best performance threshold will be the one that minimizes the confusion probability of (4). This best threshold coincides with the Ds value where homogeneous and heterogeneous pdf curves intersect (e.g., value 0.37 for window size $7 \times 7$ and contrast 2 in Fig. 4). When moving a processing window on a SAR image the user can fix the window size, but edges with many different contrasts can be found. Then the optimal threshold might be selected for each window size as the one which minimizes the confusion probability integrated over the entire range of contrasts to be considered. The confusion probability when using

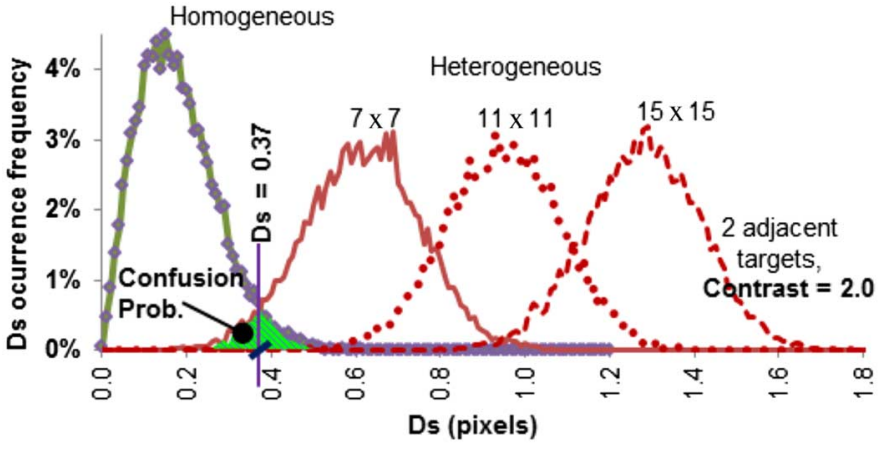

Fig. 4. Ds pdf obtained through Monte Carlo simulations of heterogeneous targets (geometry A) of contrast 2 and different window sizes $(\mathrm{ENL}=4)$.

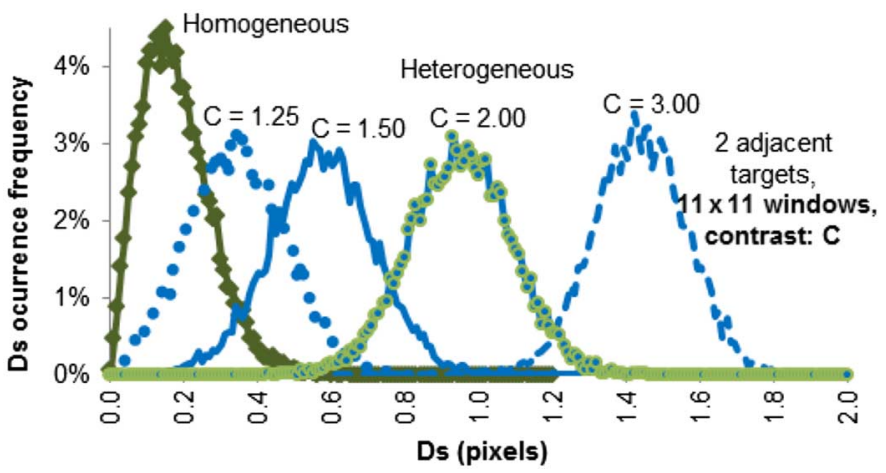

Fig. 5. Ds pdf obtained through Monte Carlo simulations of heterogeneous targets (geometry A) of different contrasts in $11 \times 11$ pixel windows $(\mathrm{ENL}=4)$.

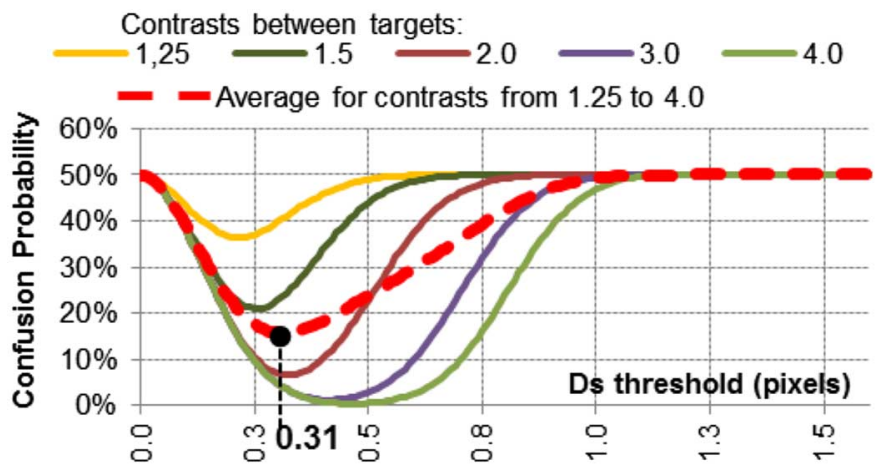

Fig. 6. Confusion probability when using the Ds operator as an edge detector, as a function of the Ds threshold used and for different contrasts between targets $(11 \times 11$ window; edge geometry A; SAR ENL $=4)$.

Ds for detecting edges in an $11 \times 11$ processing window is plotted in Fig. 6 as a function of the used Ds threshold and for different contrasts between adjacent targets. For this window size, 0.31 is the optimal threshold, i.e., the thresholds minimizing the confusion probability when contrasts between 1.25 and 4.0 are considered

$\boldsymbol{P C o n f}(T h)=\frac{\boldsymbol{P H o m}(D s \geq T h)+\boldsymbol{P} H e t(D s<T h)}{2}$.

Optimal Ds thresholds were computed for edge geometries $\mathrm{A}, \mathrm{B}, \mathrm{C}$, and $\mathrm{D}$, and for different window sizes by minimizing the integral of the confusion probability curves from contrast 1.25 to 4.0 . The confusion probability associated to 


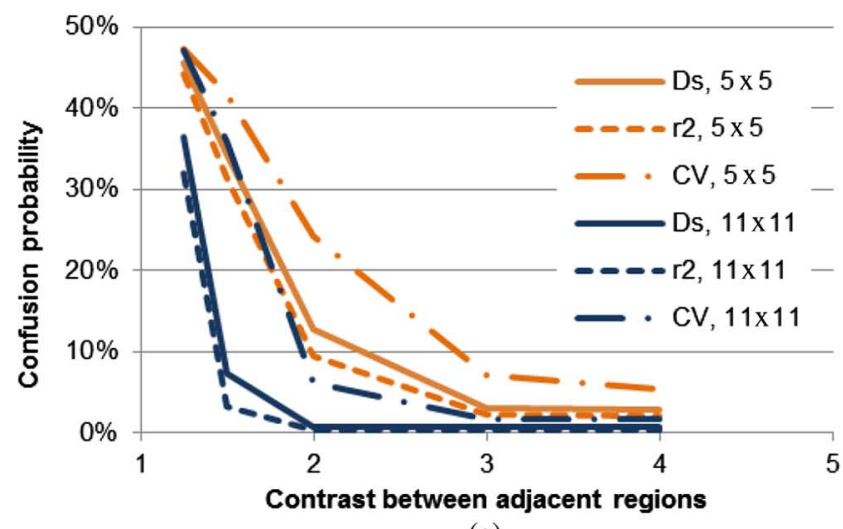

(a)

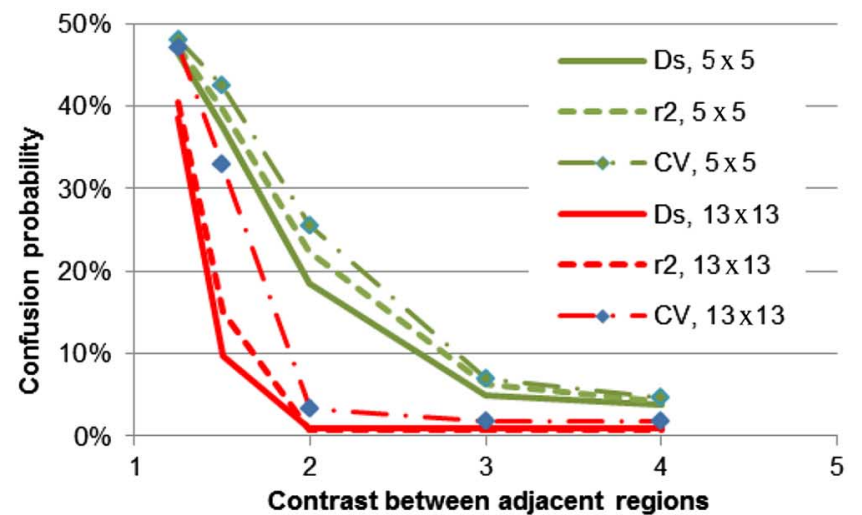

(b)

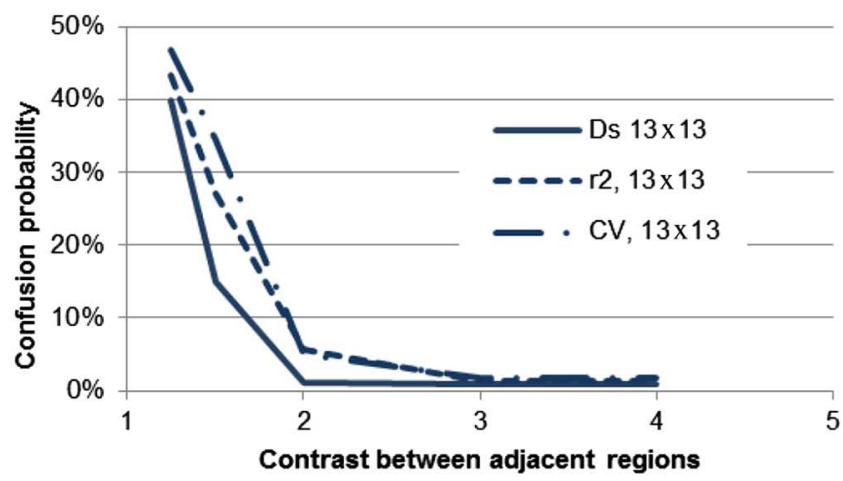

(c)

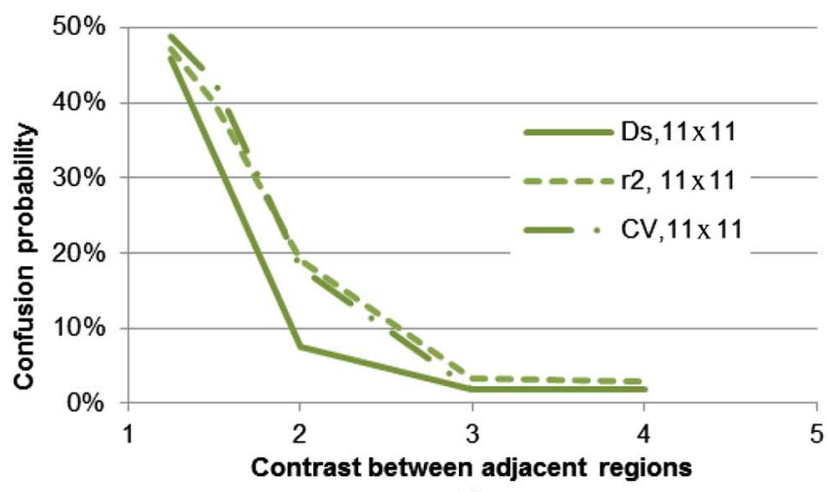

(d)

Fig. 7. Confusion probability of Ds, CV, and $\mathrm{r} 2$ for different window sizes for heterogeneous target structures: (a) geometry A; (b) geometry B; (c) geometry $\mathrm{C}$; and (d) geometry D. The confusion probabilities correspond to the optimal thresholds (ENL $=4)$. edge geometries A, B, C, and D is depicted in Fig. 7 for their corresponding optimal Ds thresholds. The confusion probability of the $\mathrm{CV}$ and the ratio edge detector $\mathrm{r} 2$, was computed in the same way as for Ds and is plotted for comparison. Note that for the computation of r2's confusion probability, the inequalities in (4) need to be inverted because $\mathrm{r} 2$ takes higher values on homogeneous windows than on heterogeneous ones.

The charts in Fig. 7 illustrate the capability of the Ds, r2, and $\mathrm{CV}$ operators to spot the presence of a boundary through the processing window center. These figures reveal that all three operators perform well at spotting edges between targets with contrast higher than 3. For lower contrasts and geometries A and $\mathrm{B}$, Ds and $\mathrm{r} 2$ show better edge sensitivity than CV. $\mathrm{r} 2$ yields slightly lower confusion probability than Ds for geometry A, though their sensitivities get closer with increasing window sizes. The relative performance of both operators is approximately inverted for geometry B. In the case of the irregular edge $\mathrm{C}$, Ds shows the lowest confusion probability. Other irregular edges were tested and yielded similar results. However, the possible irregular geometries are many, and no general conclusions are attempted for them in this paper.

Fig. 7(d) compares the capacity of Ds, r2, and CV to detect nonstationarity due to the presence of a noncentered edge (geometry D in Fig. 3). CV makes use of the one-dimensional information of the window histogram to assess stationarity, while the two-dimensional information contained in the pixel values spatial distribution, regarding the isotropy-anisotropy of the target, is omitted. Ds exploits this spatial information and, in light of Fig. 7(d), leads to lower false alarm plus missed detection rates than $\mathrm{CV}$, for edges not centered within the processing window.

\section{Use of Ds for the Assessment of Target Homogeneity in Multiresolution Filtering}

A key issue in multiresolution filtering is to determine the largest stationary window where the filtering algorithm can be applied. Implementing the Ds operator for this goal requires the use of thresholds dependent on the processing window dimension, so that the best edge presence/absence split value is used at each window size. The optimal thresholds found by minimizing the integral of the confusion probability are depicted in Fig. 8 for window sizes between $5 \times 5$ and $21 \times 21$ and different ENLs. Knowledge of the best performance threshold trends, as provided by Fig. 8, can greatly assist the selection of these values, which are critical for the quality of the results. These trends have been used in the multiresolution filtering of Doñana ASAR scenes, presented in Section III.

Gradual boundaries or transitions between targets, sketched in Fig. 3(e), can be modeled as consecutive edges of low contrast. If Ds is used on this target type to determine the largest stationary neighborhood, this will depend on the target gradient magnitude, i.e., the maximum ratio between pixel increments in value and distance on the image. This dependence is shown in Fig. 9: average Ds values of gradual boundaries are plotted as a function of window size for four different gradients. The intersections of these curves with the line of Ds thresholds show the 


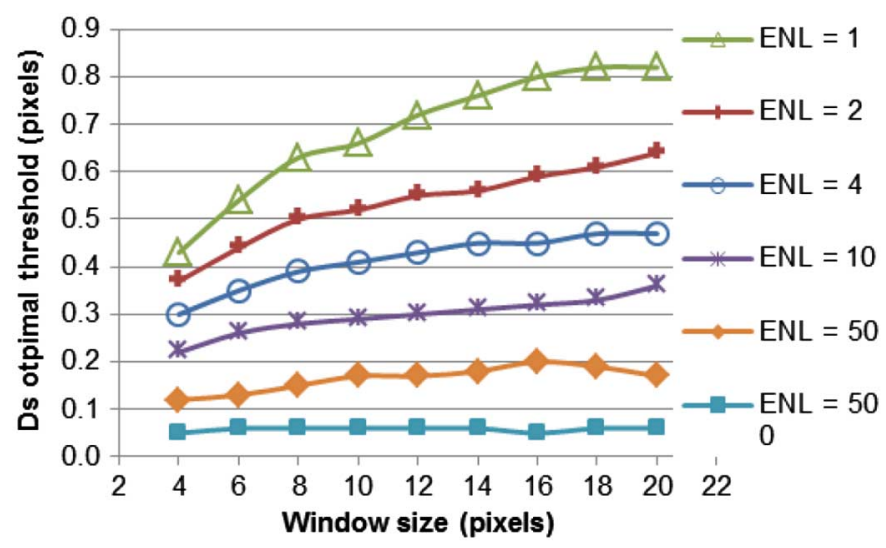

Fig. 8. Optimal Ds thresholds as a function of window size, for different ENL (geometry A).

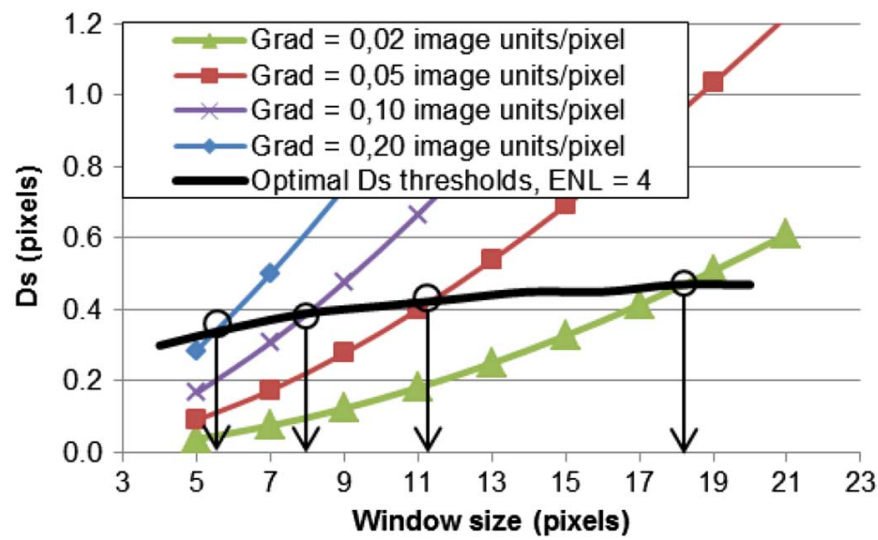

Fig. 9. Ds values of transitional targets with different gradients and maximum stationary window size for filtering purposes.

window size that would be chosen for filtering each transitional area. It can be seen that, the lower the image gradient, the larger the filtering window size.

Several authors have proposed multiresolution speckle filtering algorithms exploiting the conjunctive use of the CV and r2 operators for the detection of stationary-nonstationary state [11]-[14]. In [14], after discarding the presence of an edge through the center pixel by thresholding $\mathrm{r} 2$, significant increments in the $\mathrm{CV}$ and $\mathrm{r} 2$ values were successfully used to spot the introduction of new targets during the window expansion. The sensitivity of Ds increments for the same goal has been tested and compared to the r2s and CVs. The dots in Fig. 10 shows the average $\mathrm{Ds}, \mathrm{CV}$, and $\mathrm{r} 2$ values in a processing window incorporating two columns of a new target $\mathrm{B}$. The black lines depict the average Ds, CV, and $\mathrm{r} 2$ values for homogeneous windows. The distance of the dots to the black lines indicates the expected increment in Ds, $\mathrm{CV}$, and $\mathrm{r} 2$ caused by the introduction of a new target $\mathrm{B}$ during the filtering window expansion. As intuitively expected, $\mathrm{r} 2$ increments decrease with the window size, since the larger the processing window, the smaller the ratio represented by the new target area. Conversely, Ds increments keep increasing given that values in the periphery of the processing window contribute with a higher weight in the computation of Ds than those in the center.

\section{Use of The Ds Operator IN THE MultiResolution FILTERING OF THE DOÑANA ENVISAT/ASAR SCENES}

\section{A. Site Description}

The Doñana wetlands extent over 30000 ha on the right bank of the Guadalquivir River, near its mouth on the Atlantic Ocean coast. The wetlands undergo yearly cycles of inundation in fall and drying out in summer, with a flood extent varying considerably among years [1]. At the end of the wintertime helophyte vegetation start emerging sparsely from large part of the flooded areas. The helophytes experience rapid development during the spring season and dry out in summer. Relatively high CV's have been observed on vegetated areas in the ASAR images. This observation might be explained by the different plant developmental stage of neighboring pixels causing the target texture. As a consequence of the high CV values, some common speckle filters reduce the filtering degree and significant intensity fluctuations remain, complicating the classification of those areas as a single cover type.

In Doñana, there are few man-made structures. Edges occur in the contact between different land cover types or between flooded and emerged land. They are very often associated to the terrain contours, with capricious geometries, and normally are not as sharp as crop boundaries or roads, but show some sort of transition. Due to the terrain flatness, even the inundation perimeter often becomes a wide swamped strip.

\section{B. Imagery Data}

Numerous Envisat/ASAR scenes of the Doñana marshes were acquired from 2006 to 2010 with the main purpose to monitor the flood extent evolution [4]. The images were obtained in alternated polarization mode, with $\mathrm{HH} / \mathrm{VV}$ polarization configuration and using the seven ASAR's predetermined incidence angles or swaths [28]. The acquired data had an azimuth and range resolution of $30 \mathrm{~m}$. The image products provided by the European Space Agency had a pixel spacing of $12.5 \mathrm{~m}$ and an ENL ranging between 1.76 and 3.78. The scenes were calibrated to backscattering coefficient as explained in [4]. Implications of the incident angle and polarization configuration for the wetland observation and mapping are analyzed in [4] and [25].

\section{Filtering Methodology}

Delineation of the flooded areas from the ASAR imagery required filtering the scenes to smooth out backscattering fluctuations owing to speckle and texture within cover classes. Filtering was carried out in a multiresolution fashion and the Ds value was used to decide on the maximum homogeneous window: the processing window becomes as large as $21 \times 21$ pixels in isotropic areas and is progressively reduced when approaching edges, so that it does not ride over different land cover types. In presence of gradual boundaries, the filtering window size adapts to the gradient steepness.

The ASAR image products showed a significant spatial correlation between contiguous pixels due to the fact that the 


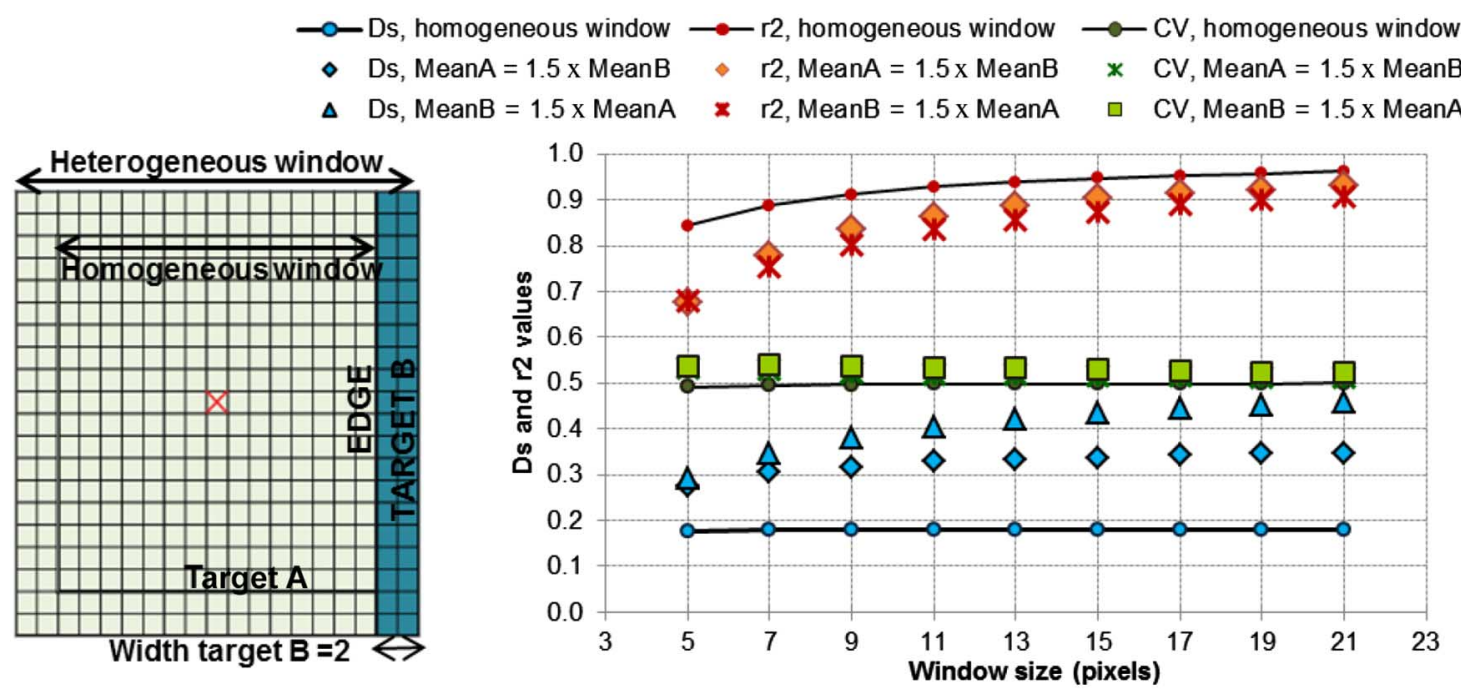

Fig. 10. Average Ds, r2, and CV values when the processing window encompasses two columns of a new target (contrast between adjacent targets: 1.5; image ENL assumed in the simulations: 4).

pixel spacing $(12.5 \mathrm{~m})$ is lower than the sensor's spatial resolution $(30 \mathrm{~m})$. The assessments and thresholds derived in Section II assumed that the pixel values were spatially independent realizations of a given statistical distribution. In order to decorrelate the ASAR pixels, new images were formed by selecting every other pixel from the original ones. Combining odd/even row with odd/even column pixels, four half size images were obtained from each ASAR one.

The Ds parameter was computed at every pixel of the four subsampled images, for odd window sizes ranging from $5 \times 5$ to $21 \times 21$. The Ds values were then placed back to their corresponding pixel's original location in the full-size ASAR image. The filtering method presented as follows used the full-size Ds and ASAR images.

The optimal set of Ds thresholds as a function of the window size was selected accordingly with the scene ENL (Fig. 8). The Ds values at each pixel $(\mathrm{i}, \mathrm{j})$ are compared to the thresholds of the corresponding window sizes $\mathrm{Th}(\mathrm{L})$, starting from $\mathrm{L} \times \mathrm{L}=5 \times 5$. If $\operatorname{Ds}(5)<\operatorname{Th}(5)$ then pixel $(\mathrm{i}, \mathrm{j})$ 's neighborhood is considered isotropic at least in a $5 \times 5$ window. For progressively increasing odd-side windows, conditions (5) and (6) below are tested. Fig. 11 assists their interpretation

$$
\begin{gathered}
D s(L+2)<T h(L+2), \text { at pixel }(i, j) \text { to be filtered } \\
D s(L)<T h(L), \text { for all pixels contiguous to }(i, j) .
\end{gathered}
$$

Only if both conditions (5) and (6) are satisfied, window $(\mathrm{L}+2) \times(\mathrm{L}+2)$ is considered isotropic and the next window size is assessed in a similar way, up to a maximum size of $21 \times 21$. If one of the above conditions is not fulfilled, then $\mathrm{L} \times \mathrm{L}$ is taken as the maximum isotropic window, which is used for filtering pixel $(\mathrm{i}, \mathrm{j})$.

An $(L+2) \times(L+2)$ window centered on pixel $(i, j)$ is portrayed in Fig. 11. Pixels contiguous to $(\mathrm{i}, \mathrm{j})$ are indicated with rings and the $\mathrm{L} \times \mathrm{L}$ subwindows for two of them are highlighted with gray and hatched backgrounds. Windows centered

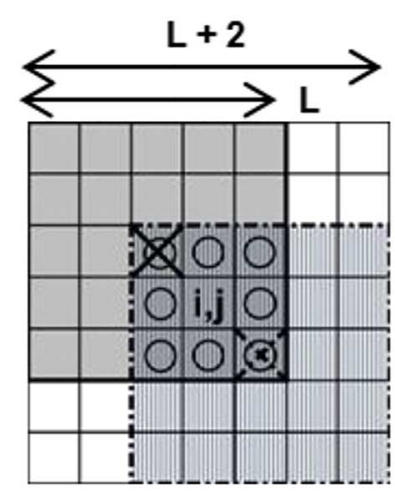

Fig. 11. Pixels contiguous to $(\mathrm{i}, \mathrm{j})$ and the $\mathrm{L} \times \mathrm{L}$ subwindows for two of them.

on symmetric features, such as curvilinear features (e.g., narrow water courses) or strong backscatterers, yield low Ds values and could be confused as isotropic neighborhoods by condition (5). Condition (6) assures that isotropy is accomplished in noncentered subwindows, preventing that sort of confusion. The presence of symmetric features could also be addressed by using higher order moments of the processing window [29]. However, specific threshold values should be computed for those moments. The contiguous pixel approach enables the use of the same set of thresholds, computed for the first moment and different window sizes.

If $\operatorname{Ds}(\mathrm{L})<\operatorname{Th}(\mathrm{L})$ is not satisfied for the starting window size $5 \times 5$, Ds is computed for all $3 \times 3$ windows which include the pixel to be filtered $(i, j)$ at any position. If the minimum resultant Ds is lower than threshold Th(3), then pixel (i,j) is filtered out using the values within the minimum Ds $3 \times 3$ window. Otherwise (i,j) is left unfiltered.

Once the maximum isotropic window had been determined following the methodology above, the pixel's filtered value was determined by simply averaging the window's intensities, since it was assumed that isotropic neighborhoods corresponded to a single cover type, the aim of the filtering was a subsequent classification and texture preservation was not a requirement. 


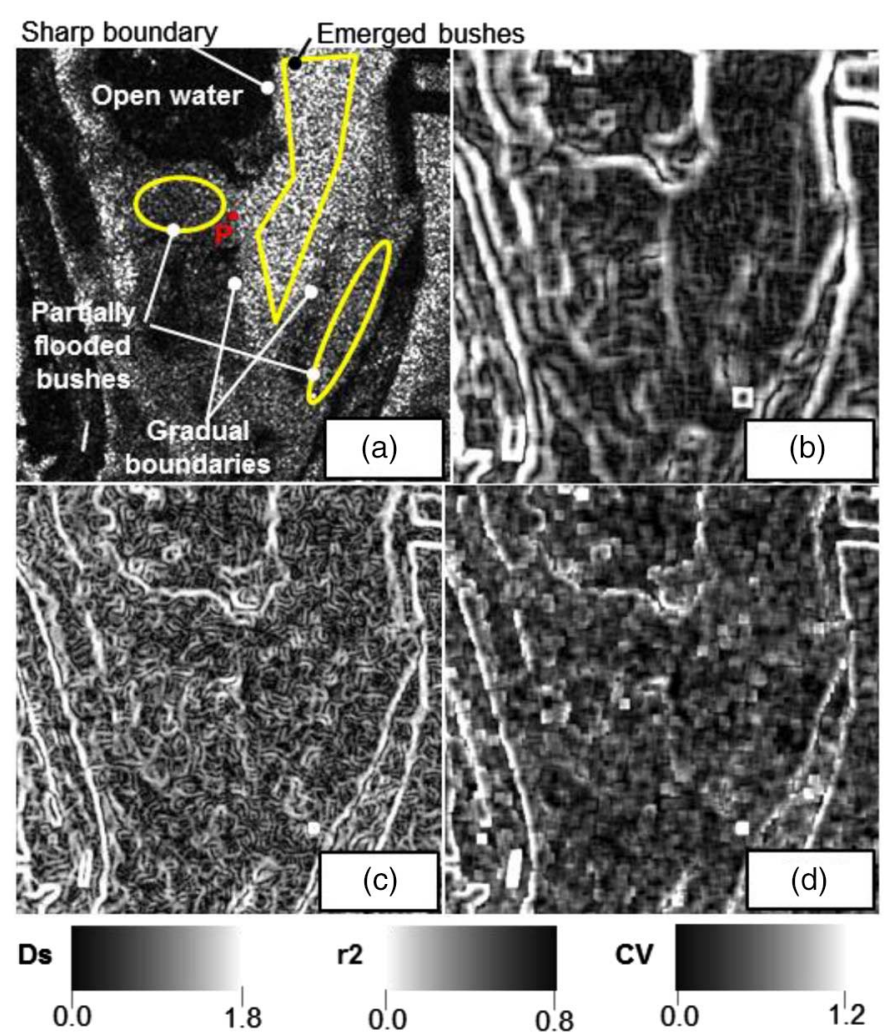

Fig. 12. Comparison among Ds, r2, and CV values: (a) fragment of the Doñana ASAR image from 21 April 2007, VV polarization, calibrated to $\sigma 0$; (b)-(d) Ds, r2, and CV values computed for the image in (a) using $9 \times 9$ processing windows and depicted as gray-scale images.

\section{Results and Discussion}

A segment of the Doñana ASAR scene captured on April 21, 2007 at $41^{\circ}$ incidence angle and VV polarization is shown in Fig. 12(a). The image includes areas of open water, plus emerged and partially flooded bushes. Sharp boundaries are seen between emerged bushes and open water surfaces, while the transition between flooded and emerged bushes is more diffuse.

Ds values computed for the image in Fig. 12(a) using $9 \times 9$ windows are depicted in Fig. 12(b). The Ds computation was performed over the de-correlated ASAR pixels, as explained in Section III-C. It can be appreciated in Fig. 12(b) that Ds is highest over the abrupt boundaries with high contrast between adjacent targets, while the lowest Ds coefficients correspond to single class areas. Gradual transitions between flooded and emerged bushes yield intermediate Ds values. Alignments indicating these transitions are discernible in Fig. 12(b). The r2 and CV coefficients obtained for Fig. 12(a) in $9 \times 9$ windows are shown in Fig. 12(c) and (d). Both, r2 and CV enable the determination of sharp edges, but the gradual ones get confused with the slightly textured natural covers. If computed over the decorrelated image, the $\mathrm{r} 2$ results approximate the Ds. However, Ds increases with the window size over gradual boundaries, while r2 keeps a stable value, as shown in Fig. 13. This Ds sensitivity enables the adaptation of the filtering window to the gradient steepness.

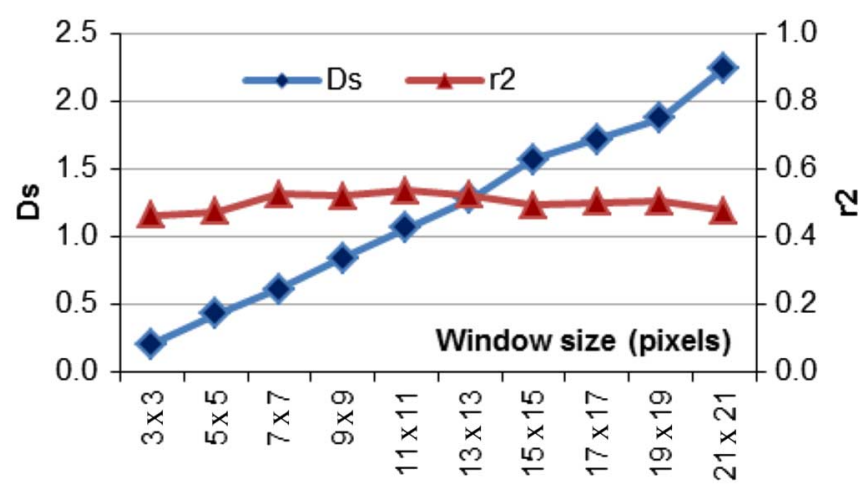

Fig. 13. Ds and $r 2$ values at point $P$ in Fig. 12(a) for different window sizes.

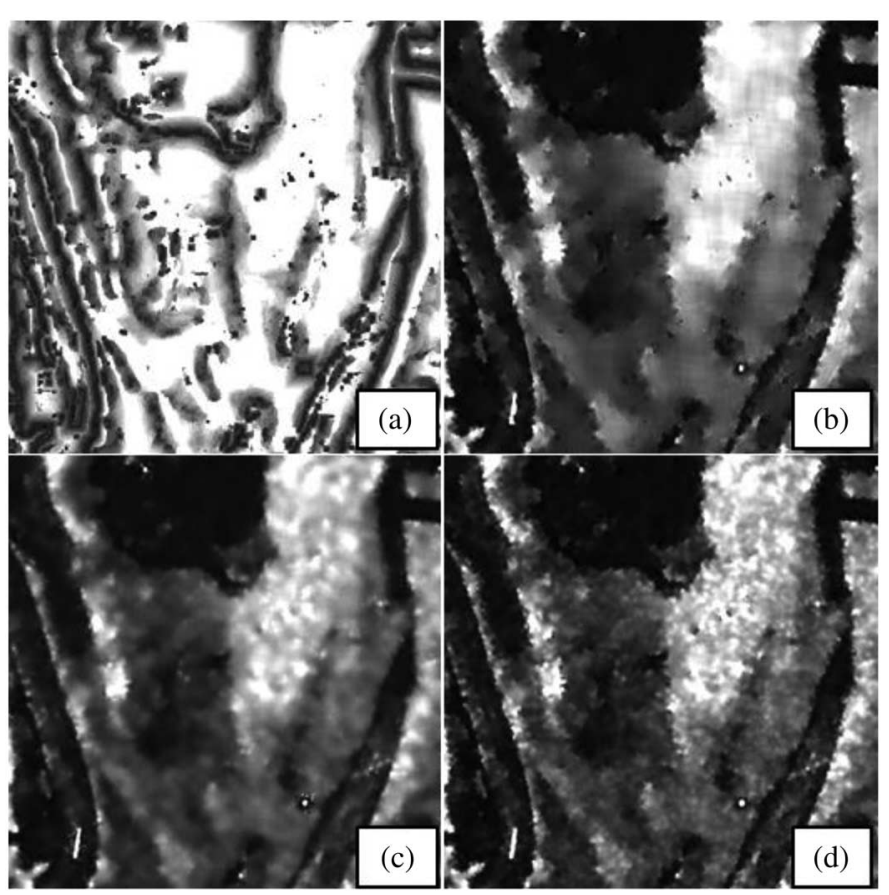

Fig. 14. Filtering of the ASAR image in Fig. 12(a): (a) maximum isotropic windows determined by Ds thresholding (from $1 \times 1$ in black to $21 \times 21$ in white); (b) image filtered by averaging within maximum isotropic windows; (c) image filtered by applying the Frost filter in $15 \times 15$ windows; (d) image filtered by applying the Frost filter within the maximum isotropic windows.

The maximum isotropic window size determined for every pixel of the ASAR image in Fig. 12(a) is shown in Fig. 14(a). The window size ranges from 1 for unfiltered pixels to 21 in the largest isotropic areas and was determined by means of the Ds parameter thresholding, as described in Section III-C. This figure reveals the adaptation of the window to the image structure: filtering windows are progressively reduced when approaching a sharp edge, intermediate sizes are adopted in the transition areas depending on the gradient steepness and the maximum size is reached over isotropic, single class regions.

The filtered ASAR image obtained by averaging within maximum isotropic windows is shown in Fig. 14(b). Fig. 14(c) and (d) shows included for comparison: they represent the results of applying the Frost filter to the ASAR image in $15 \times 15$ pixel windows and within the maximum isotropic windows, respectively. 
As it can be observed in Fig. 14, the methodology proposed in this work achieves the highest backscatter smoothing effect within cover types (open water, emerged bushes, and flooded bushes), while edges among different covers are properly preserved. The edge definition obtained by the Frost filter in Fig. 14(d) is similar to that of the proposed method in Fig. 14(b), since the same multiresolution filtering windows were used. Nevertheless, the remaining intensity fluctuations within cover types are higher after the Frost filter. This can be explained by the fact that the widely used Frost algorithm was conceived for speckle removal. Where the window's intensity $\mathrm{CV}$ is higher than that expected for a homogeneous target, as in textured cover types, the filter impulse response is reduced in order to preserve texture. As a result, the intensity fluctuations are less smoothed over textured areas even though they belong to a single class. For the specific objective of flood mapping in the Doñana wetland, texture preservation was not a requirement, while flattening intensity fluctuations within textured cover types facilitated their classification as a single category. Assuming that isotropic intensity neighborhoods correspond to a single natural cover, the Ds parameter enables the discrimination between edges and textured areas. The subsequent averaging within isotropic areas provides the highest reduction of intensity fluctuations and therefore facilitates their posterior segmentation.

Regarding the Frost algorithm applied in $15 \times 15$ pixel windows [Fig. 14(c)], it can be observed that the multiresolution filtering results in Fig. 14(b) and (c) yield a more precise edge definition of sharp edges, while the intensity smoothing degree within classes is highest in the proposed method results [Fig. 14(b)]. The Envisat/ASAR images filtered with the aid of the Ds operator were used for land cover classification and flood mapping in Doñana. The classification method is out of the scope of this paper but it can be found in [25].

It is noted that the Ds thresholds used to determine isotropy for the Doñana ASAR images were somewhat higher than the simulated ones in Fig. 8. The latter optimal thresholds were computed assuming nontextured homogeneous targets, with pixel backscattering coefficients being independent realizations of the same gamma distribution. The fact that Doñana covers exhibit texture is presumably the reason why the best thresholds turned out to be greater too. However, the thresholds satisfactorily used for filtering the Doñana images followed an increasing trend similar to that found for synthetic targets. This allowed investigating just one scaling factor for the corresponding ENL Ds trend, instead of assessing new Ds thresholds for every filtering window size.

No special treatment was given to the signals from strong scatterers. Model-based filtering algorithms generally detect the presence of such targets and preserve their signals, because they do not exhibit speckle fluctuations. In Doñana there are some metal fences and gauging station cages, both behaving as strong backscatterers [e.g., at the bottom left of Fig. 12(a)]. The location of fences and cages is precisely known, so they can be masked out and a detection algorithm of this target type is not required. However, such a detector could be easily incorporated as a first step into the presented methodology for its application to SAR images of other environments.

\section{CONCLUSION}

The Ds operator performance has been analyzed for local isotropy assessment and backscatter filtering on SAR images. It is assumed that isotropic intensity distributions in natural areas, either textured or nontextured, correspond to a single cover class. Ds is used to measure isotropy in processing neighborhoods and decide if they can be considered as belonging to a unique cover class.

Ds is computationally simple, rotationally invariant, and its calculation indicates the direction of the main intensity imbalance in the processing window. When filtering SAR images, the speckle statistical properties were introduced to determine suitable Ds thresholds for discriminating heterogeneous targets from textured cover types at different window sizes.

Simulations of the confusion probability have shown similar edge sensitivity of Ds compared to that of the $\mathrm{r} 2$ detector in presence of straight, sharp, and centered boundaries. In the case of noncentered ones, Ds performs notably better than $\mathrm{r} 2$ and $\mathrm{CV}$, which makes this operator more appropriate to spot the inclusion of heterogeneities during the processing window expansion in multiresolution filtering.

The motivation for using the Ds parameter was that some natural areas in the Doñana marshes ASAR images are textured, and significant pixel intensity fluctuations remain after applying common speckle filtering algorithms. In this study, isotropic neighborhoods were assumed to be of a single cover type and the intensities within were averaged regardless of their texture in order to flatten fluctuations and facilitate a subsequent land cover classification.

The use of Ds in a multiresolution fashion for filtering Doñana marshes ASAR scenes substantiated the usefulness of such operator. The results show the adaptation of the processing window size to the sharpness of the image structure, which is accomplished by means of the Ds thresholding; filtering windows are progressively reduced when approaching a sharp edge, intermediate sizes are adopted in the transition areas depending on the gradient gentleness, and the maximum size is reached over homogeneous regions. The homogeneity-heterogeneity Ds threshold selection for each window size was considerably simplified by using the optimal threshold trend corresponding to the scene ENL, although at least one absolute value needs to be adjusted by the user to set the scale of the trend.

\section{ACKNOWLEDGMENT}

The authors would like to express their gratitude to J. J. Egozcue from the Universitat Politècnica de Catalunya for his knowledgeable and gentle advice, to the Col-legi d'Enginyers de Camins, Canals i Ports de Catalunya for their sponsorship to Ph.D. students and to the researchers of the Espacio Natural de Doñana and the Estación Biológica de Doñana for their continuous support.

\section{REFERENCES}

[1] J. I. García, J. A. Mintegui, and J. C. Robredo, in La Vegetación en la Marisma del Parque Nacional de Doñana en Relación con su Régimen Hidráulico. Madrid, Spain: Ministerio de Medio Ambiente, 2005, pp. 30-65. 
[2] F. M. Grings et al., "Monitoring flood condition in marshes using EM models and Envisat ASAR observations," IEEE Trans. Geosci. Remote Sens., vol. 44, no. 4, pp. 936-942, Apr. 2006.

[3] F. M. Henderson and A. J. Lewis, "Radar detection of wetland ecosystems: A review," Int. J. Remote Sens., vol. 29, pp. 5809-5835, Oct. 2008.

[4] B. Marti-Cardona, C. Lopez-Martinez, J. Dolz-Ripolles, and E. BladèCastellet, "ASAR polarimetric, multi-incidence angle and multitemporal characterization of Doñana wetlands for flood extent monitoring," Remote Sens. Environ., vol. 114, no. 11, pp. 2802-2815, Nov. 2010.

[5] J. S. Lee, "Digital image enhancement and noise filtering by use of local statistics," IEEE Trans. Pattern Anal. Mach. Intell., vol. PAMI-2, no. 2, pp. 165-168, Mar. 1980.

[6] V. S. Frost, J. A. Stiles, K. S. Shanmugan, and J. C. Holtzman, "A model for radar images and its application to adaptive digital filtering of multiplicative noise," IEEE Trans. Pattern Anal. Mach. Intell., vol. PAMI-4, no. 5, pp. 157-166, Mar. 1982.

[7] D. T. Kuan, A. A. Sawchuk, T. C. Strand, and P. Chavel, "Adaptive noise smoothing filter for images with signal-dependent noise," IEEE Trans. Pattern Anal. Mach. Intell., vol. PAMI-2, no. 2, pp. 165-177, Mar. 1985.

[8] J. W. Goodman, "Statistical properties of laser speckle patterns," in Laser Speckle and Related Phenomena. Berlin, Germany: Springer-Verlag, 1975, pp. 9-75.

[9] J. S. Lee, "Refined filtering of image noise using local statistic," Comput. Graph. Image Process., vol. 15, no. 4, pp. 380-389, Apr. 1981.

[10] R. Touzi, A. Lopes, and P. Bousquet, "A statistical and geometrical edge detector for SAR images," IEEE Trans. Geosci. Remote Sens., vol. 26 no. 6, pp. 764-773, Nov. 1988.

[11] A. Lopes, R. Touzi, and E. Nezry, "Adaptive speckle filters and scene heterogeneity," IEEE Trans. Geosci. Remote Sens., vol. 28, no. 6, pp. 992 1000, Nov. 1990.

[12] Y. L. Desnos and V. Matteini, "Review on structure detection and speckle filtering on ERS-1 images," EARSeL Adv. Rem. Sen., vol. 2, no. 2, pp. 5265, 1993.

[13] A. Lopes, E. Nezry, R. Touzi, and H. Laur, "Structure detection and statistical adaptive speckle filtering in SAR images," Int. J. Remote Sens., vol. 14, pp. 1735-1758, 1993.

[14] R. Touzi, "A review of speckle filtering in the context of estimation theory," IEEE Trans. Geosci. Remote Sens., vol. 40, no. 1, pp. 2392-2404, Nov. 2002.

[15] A. Baraldi and F. Parmiggiani, "A refined gamma MAP SAR speckle filter with improved geometrical adaptivity," IEEE Trans. Geosci. Remote Sens., vol. 33, no. 5, pp. 1245-1257, Sep. 1995.

[16] Y. Yu and S. T. Acton, "Speckle reducing anisotropic diffusion," IEEE Trans. Image Process., vol. 11, no. 11, pp. 1260-1270, Jan. 2002.

[17] H. M. Salinas and D. C. Fernández, "Comparison of PDE-based nonlinear diffusion approaches for image enhancement and denoising in optical coherence tomography," IEEE Trans. Med. Imag., vol. 26, no. 6, pp. 761-771, Jun. 2007.

[18] G. Horgan, "Wavelets for SAR image smoothing," in Photogramm. Eng. Remote Sens., vol. 64, no. 12, pp. 1171-1177, Dec. 1998.

[19] H. Xie, L. E. Pierce, and F. T. Ulaby, "SAR speckle reduction using wavelet de-noising and Markov random field modeling," IEEE Trans. Geosci. Remote Sens., vol. 40, no. 10, pp. 2196-2212, Oct. 2002.

[20] B. Hou, X. Zhang, X. Bu, and H. Feng, "SAR image despeckling based on non-subsampled shearlet transform," IEEE J. Sel. Topics Appl. Earth Observ. Remote Sens., vol. 5, no. 3, pp. 809-823, Jun. 2012.

[21] S. Q. Liu, S. H. Hu, Y. Xiao, and Y. L. An, "Bayesian Shearlet shrinkage for SAR image de-noising via sparse representation," Multidimens. Syst. Signal Process., vol. 25, no. 4, pp. 683-701, Feb. 2013.

[22] D. Gleich, "Markov random field models for non-quadratic regularization of complex SAR Images," IEEE J. Sel. Topics Appl. Earth Observ. Remote Sens., vol. 5, no. 3, pp. 952-961, Jun. 2012.

[23] M. Walessa and M. Datcu, "Model-based despeckling and information extraction from SAR images," IEEE Trans. Geosci. Remote Sens., vol. 38, no. 5, pp. 2258-2269, Sep. 2000.

[24] B. Martí-Cardona, C. López-Martínez, and J. Dolz, "Local texture stationarity indicator for filtering Doñana wetlands SAR images," in Proc. Geosci. Remote Sens. Symp. (IGARSS'12), Munich, Germany, 2012, pp. 4903-4906.

[25] B. Martí-Cardona, J. Dolz-Ripollés, and C. López-Martínez, "Wetland inundation monitoring by the synergistic use of ENVISAT/ASAR imagery and ancilliary spatial data," Remote Sens. Environ., vol. 139, no. 12, pp. 171-184, Dec. 2013.
[26] A. Ramos-Fuertes, B. Martí-Cardona, E. Bladé, and J. Dolz, "Envisat/ASAR images for the calibration of the wind drag action in Doñana wetlands 2D hydrodynamic model," Remote Sens., vol. 6, no. 1, pp. 379-406, Dec. 2013.

[27] C. Oliver and S. Quegan, "Fundamental properties of SAR images," in Understanding Synthetic Aperture Radar Images. Raleigh, NC, USA: SciTech Publishing Inc., 2004, pp. 84-92.

[28] European Space Agency, ASAR Product Handbook. ESA, 2006 [Online]. Available: https://earth.esa.int/pub/ESA_DOC/ENVISAT/ASAR/asar.Pro ductHandbook.2_2.pdf

[29] M. R. Teague, "Image analysis via the general theory of moments," $J$ Opt. Soc. Amer., vol. 70, pp. 920-930, Aug. 1980.

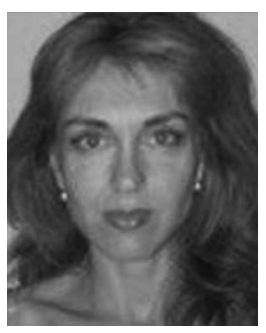

Belén Martí-Cardona received the degree in civil engineering from the Universitat Politècnica de Catalunya (UPC), Barcelona, Spain, in 1997. She received the M.Sc. degree in water resources management and remote sensing from the University of California, Davis, CA, USA, in 2004. She received the Ph.D. degree in SAR satellite data for wetland mapping from UPC in 2014.

She worked in the design and construction of hydraulic and environmental infrastructures, and in fluid dynamics numerical modeling and flood mapping in Spain and U.K. Currently, she is a Researcher with the Hydraulic, Maritime, and Environmental Engineering Department, UPC. Her research interests include development, application, and teaching of remote sensing methods for water resources and environmental modeling and management, with a special focus on wetland conservation.

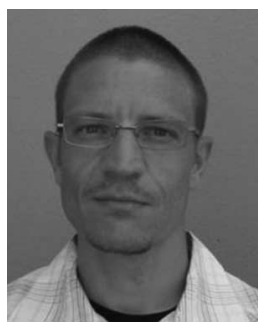

Carlos López-Martínez received the M.Sc. degree in electrical engineering and the Ph.D. degree in SAR multidimensional speckle noise modelling and filtering from the Universitat Politècnica de Catalunya, Barcelona, Spain, in 1999 and 2003, respectively.

From October 2000 to March 2002, he was with the Frequency and Radar Systems Department, HR, German Aerospace Center, DLR, Oberpfaffenhofen, Germany. From June 2003 to December 2005, he was with the Image and Remote Sensing Group, SAPHIR Team, Institute of Electronics and Telecommunications of Rennes (I.E.T.R.-CNRS UMR 6164), Rennes, France. In January 2006, he joined as a Ramón-y-Cajal Researcher with the Universitat Poltècnica de Catalunya, where he is currently an Associate Professor in the area of remote sensing and microwave technology. His research interests include SAR and multidimensional SAR, radar polarimetry, physical parameter inversion, digital signal processing, estimation theory, and harmonic analysis. He has organized different invited sessions in international conferences on radar and SAR polarimetry. He has presented advanced courses and seminars on radar polarimetry to a wide range of organizations andlbreak events.

Dr. López-Martínez is the Associate Editor of the IEEE JOURNAL OF SELECTED TOPICS IN APPLIED EARTH OBSERVATIONS AND REMOTE SENSING and he served as a Guest Editor of the EURASIP Journal on Advances in Signal Processing. He received the Student Prize Paper Award at the EUSAR 2002 Conference, Cologne, Germany.

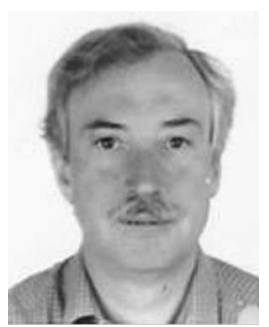

Josep Dolz-Ripollés received the degree in civil engineering from the Universidad de Santander, Santander, Spain, in 1976, and the Ph.D. degree from the Universitat Politècnica de Catalunya (UPC), Barcelona, Spain, in 1981.

Since 1989, he has been a Full Professor of Hydraulic Engineering with the UPC. Currently, he is the Director of the Flumen Institute, UPC, research organization devoted to hydrologic engineering and river dynamics modeling. 